\title{
ALGEBRA STRUCTURES ON THE COMPARISON OF THE REDUCED BAR CONSTRUCTION AND THE REDUCED $\boldsymbol{W}$-CONSTRUCTION
}

\author{
V. Álvarez, J. A. Armario, M. D. Frau, and P. Real \\ Departamento de Matemática Aplicada I, Universidad de Sevilla, \\ Sevilla, Spain
}

For a simplicial augmented algebra K, Eilenberg-Mac Lane constructed a chain map $g: B_{N}\left(K_{N}\right) \rightarrow W_{N}(K)$. They proved that $g$ is a reduction (homology isomorphism) and conjectured that it is also the injection of a contraction (special homotopy

equivalence). The contraction $C_{W-B}:\left\{W_{N}(K), B_{N}\left(K_{N}\right), f, g, \phi\right\}$ is followed at once by using homological perturbation techniques. If $K$ is commutative, Eilenberg-Mac Lane proved that $g$ is a morphism of DGAalgebras. The present article is devoted to proving that $f$ and $\phi$ satisfy certain multiplicative properties (weaker than g) and showing how they can be used for computing in an economical way the homology of twisted cartesian products of two Eilenberg-Mac Lane spaces.

Key Words: Bar and $W$-constructions; Contraction; Homological perturbation lemma; Simplicial algebras.

2000 Mathematics Subject Classification: $\quad$ 55R20; $18 \mathrm{D} 99$.

\section{INTRODUCTION}

Eilenberg-Mac Lane introduced the bar and $W$-constructions in [5] as important algebraic tools for computing the groups $H_{*}(\pi, n)$. They showed that for any simplicial augmented algebra, $K$, there is a "reduction" of the reduced normalized $W$-construction $\bar{W}_{N}(K)$ of $K$ onto the reduced normalized bar construction $\bar{B}_{N}\left(K_{N}\right)$ of the normalized chain algebra $K_{N}$ of $K$. They then raised the question whether this reduction, $g: \bar{B}_{N}\left(K_{N}\right) \rightarrow \bar{W}_{N}(K)$, is in fact part of a contraction. The homological perturbation theory answers this question by establishing such a contraction as $C_{\bar{W}-\bar{B}}:\left\{\bar{W}_{N}(K), \bar{B}_{N}\left(K_{N}\right), f, g, \phi\right\}$ (see $\left.[15,17,19]\right)$. The basic tool is the "perturbation lemma" exploited in $[8,9]$.

If the algebra $K$ is commutative, it is well known that $\bar{W}_{N}(K)$ and $\bar{B}_{N}\left(K_{N}\right)$ are commutative DGA-algebras as well. Although Eilenberg-Mac Lane showed that $g$ is a DGA-algebra morphism, nothing is known so far about the multiplicative behavior of the other morphisms that compose the contraction between $\overline{W_{N}}(K)$ and

Address correspondence to J. A. Armario, Departamento de Matemática Aplicada I, ETSII, Universidad de Sevilla, Avda. Reina Mercedes, Sevilla, S.N. 41012, Spain; Fax: +34-954557878. E-mail: armario@us.es 
$\bar{B}_{N}\left(K_{N}\right)$ (the projection $f$ and the homotopy operator $\phi$ ). Taking as a starting point Real's work in [15], we prove here that $f$ and $\phi$ are a "quasi algebra" projection and a "quasi algebra" homotopy, respectively. The result, apart from being interesting in its own right, provides a step towards designing "economical" algorithms for computing the homology of twisted cartesian products of two Eilenberg-Mac Lane spaces.

Our approach for proving the main statement of this article depends deeply upon a suitable development of the homological perturbation theory (HPT) [8, 9], particular to the category of DGA-algebras [16]. The cornerstone of HPT is the Basic Perturbation Lemma (BPL), which gives an explicit formula for transferring a perturbation of the differential of a chain complex to an equivalent chain subcomplex. $[9,11]$ prove that the BPL also holds true in the category of algebra, assuming that the data has the "maximum" degree of compatibility with regards to the algebra structures. [16] assumed weaker hypotheses for the data involved in the BPL, the analogous result for the algebra category was obtained wherein the resulting contraction had weaker properties as well. The result, which is essential in this article, is stated in Theorem 4.1.

The article is organized as follows. Section 2 describes the main algebraic and simplicial objects we intend to deal with, it also contains the objects studied in the homological perturbation theory. Section 3 is devoted to the main ideas of Real's work [15] on the construction of the contraction $C_{\bar{W}-\bar{B}}$ from $\bar{W}_{N}(K)$ to $\bar{B}_{N}\left(K_{N}\right)$ hoped for by Eilenberg-Mac Lane. In Section 4 we analyze the multiplicative behavior of the morphisms involved in the contraction $C_{\bar{W}-\bar{B}}$, under the hypothesis that $K$ is a commutative augmented simplicial algebra. This study relies heavily on the outline of the construction of $C_{\bar{W}-\bar{B}}$ given in Section 3 and uses Theorem 4.1 as a principal tool. Section 5 is devoted to describing how to use the multiplicative properties of the morphisms involved in the contraction $C_{\bar{W}-\bar{B}}$ for reducing the cost of computing the homological models of twisted cartesian products of two Eilenberg-Mac Lane spaces.

\section{PRELIMINARIES}

We will quickly review some basic notions on homological algebra specific to HPT, and introduce the notation and terminology used throughout the remainder of this article. More details can be found in [13]. Let $\Lambda$ be a commutative ring with nonzero unit, taken henceforth as ground ring and fixed throughout, and $A$ be an augmented differential graded algebra over $\Lambda$, briefly DGA-algebra. The differential, product, augmentation, and coaugmentation of $A$ will be denoted, respectively, by $d_{A}, \mu_{A}, \epsilon_{A}$, and $\eta_{A}$. Nevertheless, we will sometimes write them simply as $d, \mu, \epsilon$, and $\eta$, when no confusion can arise. In what follows, the Koszul sign conventions will be used. A morphism $\rho: A_{*} \rightarrow A_{*-1}$ is called derivation if it is compatible with the algebra structures on $A$. The degree of an element $a \in A$ is denoted by $|a|$. If $B$ is a DGA-algebra, then $A \otimes B$ has an algebra structure by means of the canonical morphism $\mu_{A \otimes B}=\left(\mu_{A} \otimes \mu_{B}\right)\left(1_{A} \otimes T \otimes 1_{B}\right)$, where $T(b \otimes a)=$ $(-1)^{|b||a|} a \otimes b$. If the DG-algebra $A$ is connected, that is $A_{0}=\Lambda$ and $d_{1}: A_{1} \rightarrow A_{0}$ is zero, then there is a canonical augmentation $\epsilon_{A}=1_{\Lambda}: A_{0} \rightarrow \Lambda$.

Here we use the normalized reduced bar construction $\bar{B}_{N}(A)$ of a DGA-algebra $A$. Recall that it is defined as the connected DGA-coalgebra, 
$\left.\bar{B}_{N}(A)=T^{c}(S(\bar{A}))\right)$, where $T^{c}()$ is the tensor coalgebra, $S()$ is the suspension functor and $\bar{A}=\operatorname{Ker} \epsilon_{A}$ is the augmentation ideal of $A$. The element on zero degree of $\bar{B}_{N}(A)$ corresponding to the identity element of $\Lambda$ is denoted by [], and the element $S \bar{a}_{1} \otimes \cdots \otimes S \bar{a}_{n}$ of $\bar{B}_{N}(A)$ is denoted by $\left[a_{1}|\cdots| a_{n}\right]$. The tensor and simplicial degrees of the element $\left[a_{1}|\cdots| a_{n}\right]$ are $\left|\left[a_{1}|\cdots| a_{n}\right]\right|_{t}=\sum\left|a_{i}\right|$ and $\left|\left[a_{1}|\cdots| a_{n}\right]\right|_{s}=n$, respectively; its total degree is the sum of its tensor and simplicial degrees. The tensor and simplicial differentials are defined by

$$
d_{t}\left(\left[a_{1}|\cdots| a_{n}\right]\right)=-\sum_{i}(-1)^{e_{i-1}}\left[a_{1}|\cdots| d_{A}\left(a_{i}\right)|\cdots| a_{n}\right]
$$

and

$$
d_{s}\left(\left[a_{1}|\cdots| a_{n}\right]\right)=\sum_{i}(-1)^{e_{i}}\left[a_{1}|\cdots| \mu_{A}\left(a_{i} \otimes a_{i+1}\right)|\cdots| a_{n}\right]
$$

where

$$
e_{i}=i+\left|a_{1}\right|+\cdots+\left|a_{i}\right|
$$

The augmentation $\varepsilon_{B(A)}: \bar{B}_{N}(A) \rightarrow \Lambda$ is determined by

$$
\varepsilon_{B(A)}([])=1, \quad \varepsilon_{B(A)}(u)=0 ; \quad \text { for } u \in \bar{B}_{q, N}(A), q>0 .
$$

If the product of $A$ is commutative, a product $*$ (called shuffle product) can be defined on $\bar{B}_{N}(A)$. In this way, the reduced bar construction has a commutative Hopf algebra structure.

Now we recall some basic concepts of simplicial topology.

A simplicial $\Lambda$-algebra (or a simplicial algebra) is a simplicial object in the category of $\Lambda$-algebras ([13, p. 233]); in other words, a simplicial algebra $K$ is a simplicial set $K=\left(K_{n}, \partial_{i}, s_{i}\right)$ where every $K_{n}$ is an algebra and every face or degeneracy operator is compatible with the algebra structures (in a similar way, a simplicial module is defined). If every $K_{n}$ is a commutative algebra, then $K$ is a commutative simplicial algebra. The inner product of two elements $a$ and $b$ of a simplicial algebra is defined only if $a$ and $b$ have the same dimension $n$, and the product is the product of $a$ and $b$ in $K_{n}$. An augmentation in a simplicial algebra $K$ is a morphism of algebras $\varepsilon: K_{0} \rightarrow \Lambda$ satisfying the relation $\varepsilon \partial_{0}=\varepsilon \partial_{1}$.

A simplicial algebra defines a differential graded algebra (DG-algebra) where the underlying graded module is the direct sum $\bigoplus_{n} K_{n}$; the product, which is called the external product, is defined by the formula

$$
a_{p} \diamond b_{q}=\sum_{(\alpha, \beta)}(-1)^{s g(\alpha, \beta)}\left(s_{\beta_{q}} \ldots s_{\beta_{1}} a_{p}\right) \cdot\left(s_{\alpha_{p}} \ldots s_{\alpha_{1}} b_{q}\right), \quad a_{p} \in K_{p}, \quad b_{q} \in K_{q} .
$$

In this formula, the pair $(\alpha, \beta)$ runs through the set of all $(p, q)$-shuffles and $\operatorname{sg}(\alpha, \beta)=\sum_{i=1}^{p}\left(\alpha_{i}-(i-1)\right)$. Finally, the differential $d$ is the boundary operator

$$
d\left(a_{p}\right)=\sum_{i=0}^{p}(-1)^{i} \partial_{i}\left(a_{p}\right), \quad a_{p} \in K_{p} .
$$


The corresponding normalized chain complex $K_{N}$ is also a DG-algebra. If the initial simplicial algebra is commutative, the induced DG-algebra is commutative as well. An augmentation of simplicial algebras induces an augmentation of DG-algebras.

Each simplicial algebra $K$ has a canonical base point $K^{u}$, a simplicial subalgebra, where $K_{n}^{u}=\Lambda$, the subalgebra generated by the unit $1_{n, K}$ of $K_{n}$, the face and degeneracy operators being the identity. An augmentation $\varepsilon: K_{0} \rightarrow \Lambda$ induces a simplicial augmentation $\varepsilon^{s}: K \rightarrow K^{u}$ defined by $\varepsilon_{n}^{s}\left(a_{n}\right)=\varepsilon\left(\partial_{0}^{n} a_{n}\right) 1_{n, K}$ if $a_{n}$ is an $n$-simplex of $K$; each $\varepsilon_{n}^{s}$ is the augmentation of the corresponding $K_{n}$. The elements of $K^{u}$ are called the scalar elements of $K_{n}$.

The kernel $\bar{K}$ of this simplicial augmentation is a simplicial submodule of $K$; each $\bar{K}_{n}$ is the augmentation ideal of $K_{n}$. This simplicial submodule is also an ideal of $K$ for the external product, that is, for the DG-algebra structure. Furthermore, $K=K^{u} \oplus \bar{K}$.

The simplicial augmented module $\bar{W}(K)$ is defined as

$$
\bar{W}_{n}(K)=K_{n-1} \otimes \cdots \otimes K_{0} \otimes \Lambda
$$

(in particular, $\bar{W}_{0}(K)=\Lambda$ ). An $n$-dimensional element $a_{n-1} \otimes \cdots \otimes a_{0} \otimes 1_{\Lambda}$ will be denoted by $\left(a_{n-1}, \ldots, a_{0}\right)$ if $n>0$ and $1_{\Lambda}$ is the unique generator of $\bar{W}_{0}(K)$.

The face and degeneracy operators of $\bar{W}(K)$ are defined as follows:

$$
\begin{aligned}
\partial_{0}\left(a_{n-1}, \ldots, a_{0}\right) & =\varepsilon^{s}\left(a_{n-1}\right)\left(a_{n-2}, \ldots, a_{0}\right), \\
\partial_{i}\left(a_{n-1}, \ldots, a_{0}\right) & =\left(\partial_{i-1} a_{n-1}, \partial_{i-2} a_{n-2}, \ldots,\left(\partial_{0} a_{n-i}\right) a_{n-i-1}, a_{n-i-2}, \ldots, a_{0}\right) \quad 0<i<n, \\
\partial_{n}\left(a_{n-1}, \ldots, a_{0}\right) & =\left(\partial_{n-1} a_{n-1}, \partial_{n-2} a_{n-2}, \ldots, \partial_{1} a_{1}\right) \varepsilon\left(a_{0}\right), \quad \text { and } \\
s_{i}\left(a_{n-1}, \ldots, a_{0}\right) & =\left(s_{i-1} a_{n-1}, \ldots, s_{0} a_{n-i}, 1_{(n-i, K)}, a_{n-i-1}, \ldots, a_{0}\right) .
\end{aligned}
$$

The augmentation of $\bar{W}(K)$ is the identity $\varepsilon_{\bar{W}}: \bar{W}_{0}(K)=\Lambda \rightarrow \Lambda$.

The simplicial module $\bar{W}(K)$, or more precisely the underlying DGA-module, carries a natural coalgebra structure with the same augmentation (or counit) and a coproduct which is defined as follows:

$$
\begin{aligned}
\Delta: \bar{W}(K) & \rightarrow \bar{W}(K) \otimes \bar{W}(K) \\
\Delta\left(a_{n-1}, \ldots, a_{0}\right) & =\sum_{i=0}^{n}\left(\hat{\partial}^{n-i} a_{n-1}, \ldots, \hat{\partial}^{n-i} a_{n-i}\right) \otimes\left(a_{n-i-1}, \ldots, a_{0}\right),
\end{aligned}
$$

where the operator $\hat{\partial}$ is the Whitney operator $\hat{\partial}(x)=\partial_{n} x$ if $x$ is $n$-dimensional and $\hat{\partial}^{n-i}=\hat{\partial} \stackrel{n-i}{n} \hat{\partial}$.

The normalization $\bar{W}_{N}(K)$ of $\bar{W}(K)$ is defined in the usual way and inherits a DGA-coalgebra structure. $\bar{W}_{n, N}(K)$ denotes the $n$ th-level of $\bar{W}_{N}(K)$ constituted by the elements of degree $n$. Let us note that if $\left(a_{n-1}, \ldots, a_{0}\right) \in \bar{W}_{n, N}(K)$, then $a_{n-1} \in \bar{K}_{n-1}$. Otherwise, this element of $\bar{W}_{n}(K)$ is in fact $s_{0}$-degenerated.

If the simplicial algebra $K$ is commutative, the (trivial) inner product:

$$
\left(a_{n-1}, \ldots, a_{0}\right) \cdot\left(b_{n-1}, \ldots, b_{0}\right)=\left(a_{n-1} b_{n-1}, \ldots, a_{0} b_{0}\right)
$$


provides $\bar{W}(K)$ with a structure of commutative simplicial algebra; indeed the commutativity property of $K$ allows this inner product to be compatible with the simplicial structure. In such a case, as explained previously, an algebra structure is also defined on $\bar{W}(K)$; this structure is compatible with the coalgebra structure, so that $\bar{W}(K)$ (and also $\bar{W}_{N}(K)$ ) becomes a Hopf algebra.

If $G$ is a simplicial monoid, and if $\Lambda[G]$ is the usual $\Lambda$-algebra of $G$, then $\bar{W}(\Lambda[G])$ is nothing but the chain complex associated to the classifying space of $G$.

Now, we define precisely the objects studied in homological perturbation theory and sketch a familiar example.

A contraction (see [5,11]) is a data set $c:\{N, M, f, g, \phi\}$ where $f: N \rightarrow M$ and $g: M \rightarrow N$ are morphisms of DG-modules (called, respectively, the projection and the inclusion), and $\phi: N \rightarrow N$ is a morphism of graded modules of degree +1 (called the homotopy operator). These data are required to satisfy the rules: (c1) $f g=1_{M}$, (c2) $\phi d_{N}+d_{N} \phi+g f=1_{N}$, (c3) $\phi \phi=0$, (c4) $\phi g=0$, and (c5) $f \phi=0$. These last three are called the side conditions [12]. In fact, these may always be assumed to hold, since the homotopy $\phi$ can be altered to satisfy these conditions [8]. These formulas imply that both chain complexes $N$ and $M$ have the same homology. We will also denote a contraction $c$ by $(f, g, \phi): N \Rightarrow M$.

The Eilenberg-Zilber theorem [7] provides the most classic example of a contraction of chain complexes. An Eilenberg-Zilber contraction is defined in [6] by the data set

$$
E Z_{F, B}:\left\{C_{N}(F \times B), C_{N}(F) \otimes C_{N}(B), A W_{F, B}, E M L_{F, B}, S H I_{F, B}\right\}
$$

where $F$ and $B$ are simplicial sets. The results of the present article depend heavily on these morphisms. We follow May's book [14] for the terminology and notation on Simplicial Topology.

We recall several results about constructions of contractions that will be used later. If we have two contractions $\left(f_{i}, g_{i}, \phi_{i}\right)$ from $N_{i}$ to $M_{i}$, for $i=1,2$ then, the following contractions can be constructed (see [7]):

i) The suspension contraction $\left(f_{i}, g_{i},-\phi_{i}\right)$ from $S\left(N_{i}\right)$ to $S\left(M_{i}\right)$;

ii) The direct sum contraction $\left(f_{1} \oplus f_{2}, g_{1} \oplus g_{2}, \phi_{1} \oplus \phi_{2}\right)$ from $N_{1} \oplus N_{2}$ to $M_{1} \oplus M_{2}$;

iii) The tensor product contraction $\left(f_{1} \otimes f_{2}, g_{1} \otimes g_{2}, \phi_{1} \otimes g_{2} f_{2}+1_{N_{1}} \otimes \phi_{2}\right)$ from $N_{1} \otimes N_{2}$ to $M_{1} \otimes M_{2}$

iv) If $N_{2}=M_{1}$, the composition contraction $\left(f_{2} f_{1}, g_{1} g_{2}, \phi_{1}+g_{1} \phi_{2} f_{1}\right)$ from $N_{1}$ to $M_{2}$.

One of the cornerstones of the homological perturbation theory is the Basic Perturbation Lemma. It provides a beautiful way of unifying many disparate results in Algebraic Topology concerning chain homotopy equivalences, and it can be used to find new results as well.

Now, we recall the concept of a perturbation datum. Let $N$ be a graded module, and let $f: N \rightarrow N$ be a morphism of graded modules. The morphism $f$ is pointwise nilpotent if for all $x \in N(x \neq 0)$, a positive integer $n$ exists (in general, the number $n$ depends on the element $x$ ) such that $f^{n}(x)=0$. A perturbation of a $D G$-module $N$ is a morphism of graded modules $\delta: N \rightarrow N$ of degree -1 , such that $\left(d_{N}+\delta\right)^{2}=0$ and $\delta_{1}=0$. A perturbation datum of the contraction $c:\{N, M, f, g, \phi\}$ 
is a perturbation $\delta$ of the DGA-module $N$ verifying that the composition $\phi \delta$ is pointwise nilpotent.

A Transference Problem consists of a contraction $c:\{M, N, f, g, \phi\}$ together with a perturbation $\delta$ of the DG-module $N$. The problem is to determine new morphisms $d_{\delta}, f_{\delta}, g_{\delta}$, and $\phi_{\delta}$ such that $c_{\delta}:\left\{\left(N, d_{N}+\delta\right),\left(M, d_{M}+d_{\delta}\right), f_{\delta}, g_{\delta}, \phi_{\delta}\right\}$ is a contraction.

The Basic Perturbation Lemma $([3,8,9,16])$ gives an explicit solution to the Transference Problem, assuming that $\delta$ is a perturbation datum of $c$.

Theorem 2.1 (BPL). Let $c:\{N, M, f, g, \phi\}$ be a contraction and $\delta: N \rightarrow N$ a perturbation datum of $c$. Then, a new contraction

$$
c_{\delta}:\left\{\left(N, d_{N}+\delta\right),\left(M, d_{M}+d_{\delta}\right), f_{\delta}, g_{\delta}, \phi_{\delta}\right\}
$$

is defined by the formulas: $d_{\delta}=f \delta \Sigma_{c}^{\delta} g, f_{\delta}=f\left(1-\delta \Sigma_{c}^{\delta} \phi\right), g_{\delta}=\Sigma_{c}^{\delta} g, \phi_{\delta}=\Sigma_{c}^{\delta} \phi$, where

$$
\Sigma_{c}^{\delta}=\sum_{i \geq 0}(-1)^{i}(\phi \delta)^{i}=1-\phi \delta+\phi \delta \phi \delta-\cdots+(-1)^{i}(\phi \delta)^{i}+\cdots
$$

Let us note that $\Sigma_{c}^{\delta}(x)$ is a finite sum for each $x \in N$, because of the pointwise nilpotency of the composition $\phi \delta$. Moreover, it is obvious that the morphism $d_{\delta}$ is a perturbation of the DG-module $\left(M, d_{M}\right)$.

The twisted Eilenberg-Zilber theorem can be seen as an important example of the usefulness of this lemma (see [18]). It solves the Transference Problem for twisted cartesian products. In the sequel, we give the ingredients for a concrete statement of this result.

Consider two simplicial sets $F, B$ and a simplicial group $G$ which operates on $F$ from the left. A twisted cartesian product $E$ with fibre $F$, base $B$, and structural group $G$ consists of a simplicial set $E_{n}=F_{n} \times B_{n}$ and

$$
\begin{aligned}
\partial_{0}(f, b) & =\left(\tau b \star \partial_{0} f, \partial_{0} b\right) \\
\partial_{i}(f, b) & =\left(\partial_{i} f, \partial_{i} b\right), \quad \text { for } i>0 \\
s_{i}(f, b) & =\left(s_{i} f, s_{i} b\right), \quad \text { for } i \geq 0,
\end{aligned}
$$

as face and degeneracy operators. Here $\star: G \times F \rightarrow F$ is the action of $G$ on $F$ and $\tau$ is a twisting function, i.e., $\tau_{n}: B_{n} \rightarrow G_{n-1}, n \geq 1$ satisfies

$$
\begin{aligned}
& \partial_{0} \tau(b)=\left[\tau\left(\partial_{0} b\right)\right]^{-1} \cdot \tau\left(\partial_{1} b\right) \\
& \partial_{i} \tau(b)=\tau\left(\partial_{i+1} b\right), \quad \text { for } i>0 \\
& s_{i} \tau(b)=\tau\left(s_{i+1} b\right), \quad \text { for } i \geq 0 \\
& \tau\left(s_{0} b\right)=e_{n},
\end{aligned}
$$

where $e_{n}$ denotes the identity element of the corresponding group $G_{n}$. We write $E=F \times{ }_{\tau} B$. 
Theorem 2.2 (Twisted Eilenberg-Zilber Theorem)[18]. The morphism

$$
\delta(f, b)=\left(\tau b \star \partial_{0} f, \partial_{0} b\right)-\left(\partial_{0} f, \partial_{0} b\right), \quad(f, b) \in C_{N}(F \times B)
$$

is a perturbation datum of the contraction,

$$
E Z_{F, B}:\left\{C_{N}(F \times B), C_{N}(F) \otimes C_{N}(B), A W_{F, B}, E M L_{F, B}, S H I_{F, B}\right\}
$$

From these data, a new contraction (called the twisted Eilenberg-Zilber contraction) is obtained by applying BPL

$$
T E Z_{F, B}:\left\{C_{N}\left(F \times_{\tau} B\right),\left(C_{N}(F) \otimes C_{N}(B), d^{\otimes}+d_{\delta}\right),\left(A W_{F, B}\right)_{\delta},\left(E M L_{F, B}\right)_{\delta},\left(S H I_{F, B}\right)_{\delta}\right\},
$$

where the bigger chain complex is associated to the Twisted Cartesian Product $F \times_{\tau} B$ with fibre $F$, base $B$, and structural group $G$. And the smaller one consists of a twisted tensor product along the twisting cochain $t, C_{N}(F) \otimes_{t} C_{N}(B)$, for $t=p \circ d_{\delta} \circ \rho$

$$
C_{N}(B) \stackrel{\rho}{\longrightarrow} C_{N}(G) \otimes C_{N}(B) \stackrel{d_{\delta}}{\longrightarrow} C_{N}(G) \otimes C_{N}(B) \stackrel{p}{\longrightarrow} C_{N}(G)
$$

where

$$
\rho(x)=e_{0} \otimes x, \quad e_{0} \text { being the identity element of } G_{0} \text { and } p(y \otimes x)=y \cdot \epsilon_{C_{N}(B)} x .
$$

Hence,

$$
d_{\delta}=t \cap=\left(\mu_{C_{N}(F)} \otimes 1\right)(1 \otimes t \otimes 1)\left(1 \otimes \Delta_{C_{N}(B)}\right),
$$

where $\mu_{C_{N}(F)}$ is the module action induced by the the action $\star: G \times F \rightarrow F$.

\section{A CONTRACTION BETWEEN $\bar{W}_{N}(K)$ AND $\bar{B}_{N}\left(K_{N}\right)$}

We outline the construction of a contraction between $\bar{W}_{N}(K)$ and $\bar{B}_{N}\left(K_{N}\right)$ given in [15] based on Eilenberg-Mac Lane's work in [5]. To this end, we recall some properties on the reduced bar and reduced $W$-constructions extracted from [5]. In this section, $K$ is a given simplicial augmented algebra.

The decomposition $K=K^{u} \oplus \bar{K}$ shows that $\bar{W}(K)$ is spanned by elements $\left(a_{n-1}, \ldots, a_{0}\right)$ wherein each entry $a_{i}$ is either an identity or an element of $\bar{K}$. The simplicial submodule $\bar{W}^{(k)}(K)$ is spanned by all such elements with at most $k$ "nontrivial" entries. We thus have the family of simplicial submodules

$$
\bar{W}^{(0)}(K) \subset \cdots \subset \bar{W}^{(k)}(K) \subset \cdots \subset \bar{W}(K)
$$

and the quotients

$$
\widetilde{W}^{(k)}(K)=\frac{\bar{W}^{(k)}(K)}{\bar{W}^{(k-1)}(K)}
$$


which are simplicial submodules. Normalizing the above family, we have the following filtration of the normalized complex $\bar{W}_{N}(K)$

$$
\bar{W}_{N}^{(0)}(K) \subset \cdots \subset \bar{W}_{N}^{(k)}(K) \subset \cdots \subset \bar{W}_{N}(K) .
$$

We also have the quotients,

$$
\widetilde{W}_{N}^{(k)}(K)=\frac{\bar{W}_{N}^{(k)}(K)}{\bar{W}_{N}^{(k-1)}(K)}
$$

which coincide with the DG-modules obtained by normalizing the simplicial modules $\widetilde{W}^{(k)}(K)$.

The DGA-module $\bar{B}_{N}\left(K_{N}\right)$ carries a filtration defined by the simplicial degree. The underlying module of the $k$ th element, $\bar{B}_{N}^{(k)}\left(K_{N}\right)$, of this filtration is the direct $\operatorname{sum} \bigoplus_{i=0}^{k} \bar{K}_{N}^{\otimes i}$

$$
\bar{B}_{N}^{(0)}\left(K_{N}\right) \subset \cdots \subset \bar{B}_{N}^{(k)}\left(K_{N}\right) \subset \cdots \subset \bar{B}_{N}\left(K_{N}\right)
$$

Denote the successive quotients

$$
\widetilde{B}_{N}^{(k)}\left(K_{N}\right)=\frac{\bar{B}_{N}^{(k)}\left(K_{N}\right)}{\bar{B}_{N}^{(k-1)}\left(K_{N}\right)}
$$

which are DG-modules with underlying modules $\bar{K}_{N}^{\otimes k}$. The differential operator is that which is induced by the residual tensor differential of the bar construction.

The following lemma gives a recursive description of the complexes $\widetilde{W}_{N}^{k+1}(K)$ and $\widetilde{B}_{N}^{k+1}\left(K_{N}\right)$, which will be used extensively in the sequel.

Lemma 3.1 [5]. There are two DG-modules isomorphisms:

$$
\begin{aligned}
\varphi: \widetilde{W}_{N}^{k+1}(K) & \rightarrow S\left(\left(\bar{K} \times \widetilde{W}^{(k)}(K)\right)_{N}\right) \text { defined } \\
\text { by } \varphi\left(a_{q} \otimes u\right) & =p\left(a_{q}\right) \times u, \text { where } a_{q} \otimes u \in \widetilde{W}_{N}^{k+1}(K),
\end{aligned}
$$

and

$$
\begin{aligned}
\psi: \widetilde{B}_{N}^{k+1}\left(K_{N}\right) & \rightarrow S\left(\bar{K}_{N} \otimes \widetilde{B}_{N}^{(K)}\right) \text { defined } \\
\text { by } \psi([a \mid u]) & =p(a) \otimes u, \text { where } a \in K_{N}, \text { and } u \in \bar{B}_{N}\left(K_{N}\right)
\end{aligned}
$$

The morphism $p: K \rightarrow \bar{K}$ is defined as

$$
p\left(a_{l}\right)=a_{l}-\varepsilon^{s}\left(a_{l}\right) ; \quad a_{l} \in K_{l} .
$$

From Eilenberg-Mac Lane's work [5] on the reduction $g: \bar{B}_{N}\left(K_{N}\right) \rightarrow \bar{W}_{N}(K)$, it follows at once that, for each $k \geq 0$ a contraction, $r^{(k)}:\left(\tilde{f}_{k}, \tilde{g}_{k}, \widetilde{\phi}_{k}\right)$ from $\widetilde{W}_{N}^{(k)}(K)$ into $\widetilde{B}_{N}^{(k)}\left(K_{N}\right)$ can be constructed. In the sequel, we only show a sketch of this construction. 
Both $\widetilde{B}_{N}^{(0)}\left(K_{N}\right)$ and $\widetilde{W}_{N}^{(0)}(K)$ are the complex consisting of the ground ring in dimension 0 . Hence we have the stated result for $k=0$. For $k=1$, again we have a DG-module isomorphism where

$$
\begin{aligned}
\tilde{f}_{1}\left(a_{q} \otimes 1_{q, \bar{W}}\right) & =\left[a_{q}\right] \quad \text { if } a_{q} \otimes 1_{q, \bar{W}} \in \widetilde{W}_{q+1, N}^{(1)}(K), \\
\tilde{g}_{1}\left[a_{q}\right] & =a_{q} \otimes 1_{q, \widetilde{W}} \quad \text { if }\left[a_{q}\right] \in \widetilde{B}_{q+1, N}^{(1)}\left(K_{N}\right) \quad \text { and } \quad \tilde{\phi}_{1}=0 .
\end{aligned}
$$

For $k>1$, we have the diagram

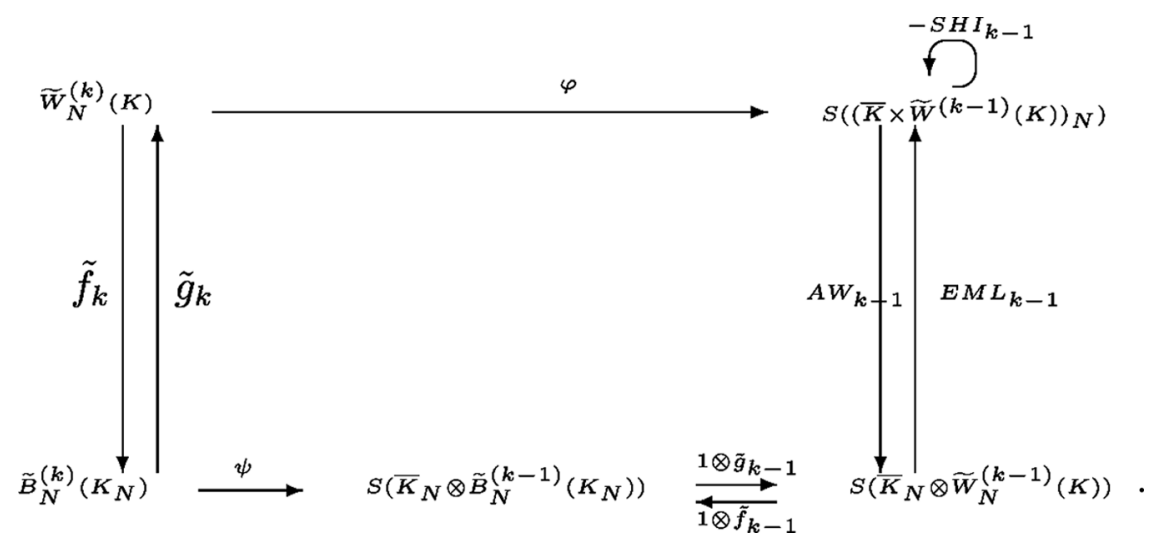

Here $\left(A W_{k-1}, E M L_{k-1}, S H I_{k-1}\right)$ are the morphisms integrating the EilenbergZilber contraction between $\left(\bar{K} \times \widetilde{W}^{(k-1)}(K)\right)_{N}$ and $\bar{K}_{N} \otimes \widetilde{W}_{N}^{k-1}(K)$. It can be proved inductively that the following data set is a contraction:

$$
r^{(k)}:\left\{\widetilde{W}_{N}^{(k)}(K), \widetilde{B}_{N}^{(k)}\left(K_{N}\right), \tilde{f}_{k}, \tilde{g}_{k}, \tilde{\phi}_{k}\right\}
$$

where

$$
\begin{aligned}
\tilde{f}_{k} & =\psi^{-1}\left(1 \otimes \tilde{f}_{k-1}\right) A W_{k-1} \varphi, \\
\tilde{g}_{k} & =\varphi^{-1} E M L_{k-1}\left(1 \otimes \tilde{g}_{k-1}\right) \psi, \\
\tilde{\phi}_{k} & =-\varphi^{-1} S H I_{k-1} \varphi-\varphi^{-1} E M L_{k-1}\left(1 \otimes \tilde{\phi}_{k-1}\right) A W_{k-1} \varphi .
\end{aligned}
$$

Thus, we can consider the direct sum of contractions

$$
\bigoplus_{k \geq 0} r^{(k)}:\left\{\bigoplus_{k \geq 0} \widetilde{W}_{N}^{(k)}(K), \bigoplus_{k \geq 0} \widetilde{B}_{N}^{(k)}\left(K_{N}\right), \bigoplus_{k \geq 0} \tilde{f}_{k}, \bigoplus_{k \geq 0} \tilde{g}_{k}, \bigoplus_{k \geq 0} \tilde{\phi}_{k}\right\}
$$

It follows at once that $\bigoplus_{k \geq 0} \tilde{g}_{k}=g$ ( $g$ is the morphism given by Eilenberg-Mac Lane).

It is clear that, from the viewpoint of the underlying graded module structure, $\bigoplus_{k \geq 0} \widetilde{W}_{N}^{(k)}(K)$ and $\bigoplus_{k \geq 0} \widetilde{B}_{N}^{(k)}\left(K_{N}\right)$ coincide with $\bar{W}_{N}(K)$ and $\bar{B}_{N}\left(K_{N}\right)$, respectively. 
Hence, given $w \in \bar{W}_{N}(K)$, there exists $k \geq 0$ such that $w \in \widetilde{W}_{N}^{(k)}(K)$. The differential operator of $\bar{W}_{N}(K)$ can be decomposed into two parts

$$
d_{\bar{W}}(w)=\rho^{k, k-1}(w) \oplus \rho^{k}(w)
$$

by the definition of the face operators. Where $\rho^{k, k-1}(w) \in \widetilde{W}_{N}^{(k-1)}(K)$ and $\rho^{k}(w) \in \widetilde{W}_{N}^{(k)}(K)$. Moreover, $\rho^{k}(w)=d_{\widetilde{W}_{N}^{(k)}}(w)$ with $d_{\widetilde{W}_{N}^{(k)}}$ as the differential in $\widetilde{W}_{N}^{(k)}$. In general,

$$
d_{\bar{W}}=\bigoplus_{k \geq 0}\left(\rho^{k, k-1}+\rho^{k}\right)
$$

and we denote,

$$
\rho=\bigoplus_{k \geq 1} \rho^{k, k-1} \quad \text { and } \quad d=\bigoplus_{k \geq 0} \rho^{k}
$$

Obviously, $d$ is the differential operator over $\bigoplus_{k \geq 0} \widetilde{W}_{N}^{(k)}(K)$ and $\rho$ a perturbation of this DG-module.

Similarly, the differential operator of $\bar{B}_{N}\left(K_{N}\right)$ is also constituted by two summands: $d_{t}$ the tensorial differential, and $d_{s}$ the simplicial differential. Clearly, $d_{t}$ is the differential operator of $\bigoplus_{k \geq 0} \widetilde{B}_{N}^{(k)}\left(K_{N}\right)$.

By a simple inspection, we have

$$
\tilde{\phi}_{k-1} \circ \rho^{k, k-1}(w) \in \widetilde{W}_{N}^{(k-1)}(K), \quad \forall w \in \widetilde{W}_{N}^{(k)}(K) .
$$

Therefore, $\rho$ is a perturbation datum of $\bigoplus_{k \geq 0} r^{(k)}$.

Using BPL with input data the contraction $\bigoplus_{k \geq 0} r^{(k)}$ and the perturbation $\rho$, it follows that

$$
\left(\bigoplus_{k \geq 0} r^{(k)}\right)_{\rho}:\left\{\bar{W}_{N}(K),\left(\bigoplus_{k \geq 0} \widetilde{B}_{N}^{(k)}\left(K_{N}\right), d_{t}+d_{\rho}\right),\left(\bigoplus_{k \geq 0} \tilde{f}_{k}\right)_{\rho},\left(\bigoplus_{k \geq 0} \tilde{g}_{k}\right)_{\rho},\left(\bigoplus_{k \geq 0} \tilde{\phi}_{k}\right)_{\rho}\right\} .
$$

It may be readily checked that

$$
\rho^{k, k-1} \tilde{g}_{k}=g_{k-1} d_{s}, \quad \forall k \geq 1,
$$

thus it follows at once that $d_{\rho}=d_{s}$ and $\left(\bigoplus_{k \geq 0} \tilde{g}_{k}\right)_{\rho}=\bigoplus_{k \geq 0} \tilde{g}_{k}$. Therefore, the desired contraction $C_{\bar{W}-\bar{B}}$, from $\bar{W}_{N}(K)$ to $\bar{B}_{N}\left(K_{N}\right)$ has been described.

\section{THE MAIN THEOREM}

In this section we assume that $K$ denotes a commutative simplicial augmented algebra. Therefore, the algebra structures are defined on $\bar{W}_{N}(K)$ and on $\bar{B}_{N}\left(K_{N}\right)$. $\nabla$ denotes the external product on $\bar{W}_{N}(K)$ and $*$ the shuffle product on $\bar{B}_{N}\left(K_{N}\right)$. These products induce an algebra structure on the complexes $\bigoplus_{k \geq 0} \widetilde{W}_{N}^{(k)}(K)$ and $\bigoplus_{k \geq 0} \widetilde{B}_{N}^{(k)}\left(K_{N}\right)$, respectively.

It may be readily verified that the following identities hold:

1. If $x^{(i)}$ denotes an element of $\widetilde{W}_{N}^{(i)}(K)$, then $x^{(p)} \nabla x^{(q)} \in \widetilde{W}_{N}^{(p+q)}(K)$; 
2. $\rho^{k} \nabla=\nabla\left(1 \otimes \rho^{k-i}+\rho^{i} \otimes 1\right), \forall k-i \geq 0, \forall i \geq 0$;

3. $\rho^{k, k-1} \nabla=\nabla\left(1 \otimes \rho^{k-i, k-i-1}+\rho^{i, i-1} \otimes 1\right), \forall k-i \geq 1, \forall i \geq 1$.

Hence the DGA-module $\bigoplus_{k \geq 0} \widetilde{W}_{N}^{(k)}(K)$ (we recall that $d=\bigoplus_{k \geq 0} \rho^{k}$ is its differential) becomes a commutative DGA-algebra with regard to the product $\nabla$. Moreover, the perturbation datum $\rho=\bigoplus_{k \geq 0} \rho^{k, k-1}$ is also a derivation.

Similar properties hold for the DGA-module $\bigoplus_{k \geq 0} \widetilde{B}_{N}^{(k)}\left(K_{N}\right)$ :

1. If $y^{(i)}$ denotes an element of $\widetilde{B}_{N}^{(i)}\left(K_{N}\right)$, then $y^{(p)} * y^{(q)} \in \widetilde{B}_{N}^{(p+q)}\left(K_{N}\right)$;

2. $d_{t} *=*\left(1 \otimes d_{t}+d_{t} \otimes 1\right)$.

Hence the DGA-module $\bigoplus_{k \geq 0} \widetilde{B}_{N}^{(k)}\left(K_{N}\right)$ (we recall that $d_{t}$ is its differential) becomes a commutative DGA-algebra with regards to the product $*$.

The goal of this section is to prove that the projection $f$ and the homotopy operator $\phi$ of the contraction $C_{\bar{W}-\bar{B}}:\left\{\bar{W}_{N}(K), \bar{B}_{N}\left(K_{N}\right), f, g, \phi\right\}$ satisfy certain multiplicative properties. For this purpose, we use some results of the homological perturbation theory extended to the category of the DGA-algebra. We begin by extending the notion of contraction to the proper category of DGA-algebra.

Let $A$ and $A^{\prime}$ be two DGA-algebras. Given a contraction $c:\left\{A, A^{\prime}, f, g, \phi\right\}$ we can distinguish different types of algebra contractions in function of the degree of compatibility of the morphisms $f, g$, and $\phi$ with regards to the products $\mu_{A}$ and $\mu_{A^{\prime}}$ (for details see [16]). We say that $c$ is:

i) A full algebra contraction if $f$ and $g$ are morphisms of DGA-algebras and $\phi$ is an algebra homotopy;

ii) An almost-full algebra contraction if $f$ and $g$ are morphisms of DGA-algebras and $\phi$ is a quasi algebra homotopy. An example of this type is the EilenbergZilber contraction (1), when $F$ and $B$ are simplicial groups;

iii) A semi-full algebra contraction if $f$ is a quasi algebra, projection, $g$ is a morphism of DGA-algebras, and $\phi$ is a quasi algebra homotopy.

Here the projection $f$ is said to be a quasi algebra projection if the following conditions hold:

$$
f \mu_{A}(\phi \otimes \phi)=0, \quad f \mu_{A}(\phi \otimes g)=0, \quad f \mu_{A}(g \otimes \phi)=0 .
$$

The homotopy operator $\phi$ is said to be a quasi algebra homotopy if the following conditions hold:

$$
\phi \mu_{A}(\phi \otimes \phi)=0, \quad \phi \mu_{A}(\phi \otimes g)=0, \quad \phi \mu_{A}(g \otimes \phi)=0 .
$$

We also recall that $\phi$ is called a homotopy algebra if

$$
\phi \mu_{A}=\mu_{A} \phi^{[\otimes 2]}
$$

where $\phi^{[\otimes 2]}=1 \otimes \phi+\phi \otimes g f$.

One can see immediately that, in the context of algebra contractions, the designation of full implies almost-full, in the same way that almost-full implies semi-full. Let us observe that each of these classes is closed under composition and 
tensor product of contractions. Examples of all these types of contractions appear in [16]. The following result tells us that the set of semi-full algebra contractions is closed under homological perturbation. This theorem is used in the proof of one of the main theorems of this article.

Theorem 4.1 (SF-APL) ([16]). Taking as input data a semi-full algebra contraction $c:\left\{A, A^{\prime}, f, g, \phi\right\}$ and an algebra perturbation datum $\delta$ of $c$, the perturbed contraction $c_{\delta}$ is a semi-full algebra contraction, where the product on $A_{\delta}^{\prime}$ is the original product $\mu_{A^{\prime}}$.

The previous result is the key which will lead us to the desired goal of this section. To this end, we need to prove the following facts:

i) The contraction $\bigoplus_{k \geq 0} r^{(k)}$ is a semi-full algebra contraction;

ii) The perturbation datum $\rho$ is a derivation.

We focus on the first one, as the second has already been proved above.

Theorem 4.2. $\bigoplus_{k \geq 0} r^{(k)}:\left\{\bigoplus_{k \geq 0} \widetilde{W}_{N}^{(k)}(K), \bigoplus_{k \geq 0} \widetilde{B}_{N}^{(k)}\left(K_{N}\right), \bigoplus_{k \geq 0} \tilde{f}_{k}, \bigoplus_{k \geq 0} \tilde{g}_{k}, \bigoplus_{k \geq 0} \tilde{\phi}_{k}\right\}$ is an almost-full algebra contraction.

Proof. From now on, we will identify both $\widetilde{W}_{N}^{(k+1)}(K)$ and $S\left(\left(\bar{K} \times \widetilde{W}^{(k)}(K)\right)_{N}\right)$ via the isomorphism $\varphi$ (see (2)). To simplify the notation, we use the same letter for referring to an element in either $\widetilde{W}_{N}^{(k+1)}(K)$ or $S\left(\left(\bar{K} \times \widetilde{W}^{(k)}(K)\right)_{N}\right)$. The context will clarify the meaning of the term when it will be necessary. In the same way, we identify in $\widetilde{B}_{N}^{(k+1)}\left(K_{N}\right)$ and $S\left(\bar{K}_{N} \otimes \widetilde{B}_{N}^{(k)}\left(K_{N}\right)\right)$ via the isomorphism $\psi($ see (3)).

Firstly, we prove that $\tilde{f}_{k}$ is a DGA-algebra morphism for each $k \geq 0$. That is,

$$
\tilde{f}_{k} \nabla=*\left(\tilde{f}_{k-i} \otimes \tilde{f}_{i}\right), \quad 0 \leq i \leq k .
$$

The proof is by induction on $k$, and is immediate for $k=0$ since $\tilde{f}_{0}$ is the identity map of $\Lambda$. Assume that the identity (5) holds for $k<n$; we now prove that it holds for $k=n$. Let $n$ be equal to $h+h^{\prime}$, where $h$ and $h^{\prime}$ denote non-negative integers, getting $a_{p} \otimes u_{p} \in \widetilde{W}_{p+1, N}^{(h)}(K)$ and $b_{q} \otimes v_{q} \in \widetilde{W}_{q+1, N}^{\left(h^{\prime}\right)}(K)$ with $a_{p} \in \bar{K}_{p}$ and $b_{q} \in \bar{K}_{q}$; it is clear that $\left(a_{p} \otimes u_{p}\right) \nabla\left(b_{q} \otimes v_{q}\right) \in \widetilde{W}_{p+q+2, N}^{\left(h+h^{\prime}\right)}(K)$.

Expanding the right term of $(5)$. We get

$$
\tilde{f}_{h}\left(a_{p} \otimes u_{p}\right) * \tilde{f}_{h^{\prime}}\left(b_{q} \otimes v_{q}\right)=\left(\left(1 \otimes \tilde{f}_{h-1}\right) A W\left(a_{p} \otimes u_{p}\right)\right) *\left(\left(1 \otimes \tilde{f}_{h^{\prime}-1}\right) A W\left(b_{q} \otimes v_{q}\right)\right)
$$

(the Alexander-Whitney operator $A W: C_{N}(F \times B) \rightarrow C_{N}(F) \otimes C_{N}(B)$ is defined by $A W\left(f_{n} \times b_{n}\right)=\sum_{i=0}^{n} \hat{\partial}^{n-i} f_{n} \otimes \partial_{0}^{i} b_{n}$, where $F$ and $B$ are simplicial sets and $\hat{\partial}$ is the last face operator and $\partial_{i}^{j}$ means $\partial_{i}{ }^{j \text { times }} \partial_{i}$ )

$$
=\left(\sum_{i=0}^{p} \hat{\partial}^{p-i} a_{p} \otimes \bar{f}_{h-1}\left(\partial_{0}^{i} u_{p}\right)\right) *\left(\sum_{j=0}^{q} \hat{\partial}^{q-j} b_{q} \otimes \bar{f}_{h^{\prime}-1}\left(\partial_{0}^{j} v_{q}\right)\right)
$$

(taking into account that each term of the above shuffle product can be considered as an element of $\widetilde{B}_{N}^{(n)}\left(K_{N}\right)$ and applying the next identity (see [5, p. 74])):

$$
[a \mid u] *[b \mid v]=[a]\left|[u *(b \mid v)]+(-1)^{|b|_{\bar{B}}|[a \mid u]|_{\bar{B}}}[b]\right|[(a \mid u) * v],
$$


where $\left.\left[a_{1}|\ldots| a_{n}\right] \mid\left[b_{1}|\ldots| b_{m}\right]=\left[a_{1}|\ldots| a_{n}\left|b_{1}\right| \ldots \mid b_{m}\right]\right)$

$$
\begin{aligned}
= & \sum_{i=0}^{p}\left[\hat{\partial}^{p-i} a_{p}\right] \mid\left[\bar{f}_{h-1}\left(\hat{\partial}_{0}^{i} u_{p}\right) * \bar{f}_{h^{\prime}}\left(b_{q} \otimes v_{q}\right)\right] \\
& +\sum_{j=0}^{q}(-1)^{(p+1)(j+1)}\left[\hat{\partial}^{q-j} b_{q}\right] \mid\left[\bar{f}_{h}\left(a_{p} \otimes u_{p}\right) * \bar{f}_{h^{\prime}-1}\left(\partial_{0}^{j} v_{q}\right)\right]
\end{aligned}
$$

(by the induction assumption)

$$
\begin{aligned}
= & \sum_{i=0}^{p}\left[\hat{\partial}^{p-i} a_{p}\right] \mid\left[\bar{f}_{h+h^{\prime}-1}\left(\partial_{0}^{i} u_{p} \nabla\left(b_{q} \otimes v_{q}\right)\right)\right] \\
& +\sum_{j=0}^{q}(-1)^{(p+1)(j+1)}\left[\hat{\partial}^{q-j} b_{q}\right] \mid\left[\bar{f}_{h+h^{\prime}-1}\left(\left(a_{p} \otimes u_{p}\right) \nabla \partial_{0}^{j} v_{q}\right)\right] .
\end{aligned}
$$

As a preliminary to the next step, it is necessary to note the following. Let $(\alpha, \beta)$ be a $(p, q)$-shuffle. If $\alpha_{1}=0$, i.e., $(\alpha, \beta)=\left(0, \alpha_{2}, \ldots, \alpha_{p} ; \beta_{1}, \ldots, \beta_{q}\right)$, then $\bar{\alpha}$ denotes the set obtained from $\alpha$ by omitting $\alpha_{1}=0$, i.e., $\bar{\alpha}=\left(\alpha_{2}, \ldots, \alpha_{p}\right)$; in an analogous way, $\bar{\beta}$ may be defined. The notation $\alpha-1$ defines the set obtained from $\alpha$ by subtracting 1 , i.e., $\alpha-1=\left(\alpha_{1}-1, \ldots, \alpha_{p}-1\right)$; the term $\beta-1$ is similarly defined.

Assuming the above notations, the succeeding assertions are satisfied [14]:

1. Let $(\alpha, \beta)$ be a $(p, q)$-shuffle, with $\alpha_{1}=0$. Then $(\bar{\alpha}-1, \beta-1)$ becomes a $(p-1, q)$-shuffle and $\operatorname{sg}(\bar{\alpha}-1, \beta-1)=\operatorname{sg}(\alpha, \beta)$. Furthermore, every $(p-1, q)$ shuffle may be constructed in this way.

2. Let $(\alpha, \beta)$ be a $(p, q)$-shuffle, with $\beta_{1}=0$. Then, $(\alpha-1, \bar{\beta}-1)$ is a $(p, q-1)$ shuffle and $\operatorname{sg}(\alpha-1, \bar{\beta}-1)=\operatorname{sg}(\alpha, \beta)+p$. Furthermore, every $(p, q-1)$-shuffle may be constructed in this way.

It is convenient to write

$$
s_{\alpha}=s_{\alpha_{p}} \ldots s_{\alpha_{1}}, \quad s_{\beta}=s_{\beta_{q}} \ldots s_{\beta_{1}} .
$$

With these notations at hand, we expand the left term of (5),

$$
\bar{f}_{h+h^{\prime}}\left(\left(a_{p} \otimes u_{p}\right) \nabla\left(b_{q} \otimes v_{q}\right)\right)=\bar{f}_{h+h^{\prime}}\left(\sum_{(\mu, v)}(-1)^{\operatorname{sg}(\mu, v)} s_{v}\left(a_{p} \otimes u_{p}\right) \cdot s_{\mu}\left(b_{q} \otimes v_{q}\right)\right),
$$

where the $(\mu, v)$ index runs over all the $(p+1, q+1)$-shuffles. Taking into account the following identities on the degeneracy operators of $\bar{W}(K)$ (see [5, p. 90]):

$$
s_{0}\left(c_{r} \otimes w_{r}\right)=1_{r+1} \otimes\left(c_{r} \otimes w_{r}\right), \quad s_{i+1}\left(c_{r} \otimes w_{r}\right)=s_{i} c_{r} \otimes s_{i} w_{r}, \quad i=0, \ldots, r
$$

with $c_{r} \in K_{r}, w_{r} \in \bar{W}_{r}(K)$. 
By (9), it is possible to rewrite (8) as

$$
\begin{aligned}
& \bar{f}_{h+h^{\prime}}\left(\sum_{\substack{(\mu, v) \\
\mu_{1}=0}}(-1)^{\operatorname{sg}(\mu, v)}\left(s_{v-1} a_{p} \otimes s_{v-1} u_{p}\right) \cdot\left(s_{\bar{\mu}}\left(1_{q+1} \otimes b_{q} \otimes v_{q}\right)\right)\right. \\
& \left.\left.\quad+\sum_{\substack{(\mu, v) \\
v_{1}=0}}(-1)^{\operatorname{sg}(\mu, v)}\left(s_{\bar{v}}\left(1_{p+1} \otimes a_{p} \otimes u_{p}\right)\right) \cdot\left(s_{\mu-1} b_{q} \otimes s_{\mu-1} v_{q}\right)\right)\right)
\end{aligned}
$$

(the first sum is taken over all $(p+1, q+1)$-shuffles $(\mu, v)$ with $\mu_{1}=0$, and the second is taken over all $(p+1, q+1)$-shuffles $(\mu, v)$ with $\left.v_{1}=0\right)$

$$
\begin{aligned}
= & \bar{f}_{h+h^{\prime}}\left(\sum_{(\bar{\mu}-1, v-1)}(-1)^{\mathrm{sg}(\bar{\mu}-1, v-1)} s_{v-1} a_{p} \otimes\left(s_{v-1} u_{p} \cdot s_{\bar{\mu}-1}\left(b_{q} \otimes v_{q}\right)\right)\right) \\
& +\bar{f}_{h+h^{\prime}}\left(\sum_{(\mu-1, \bar{v}-1)}(-1)^{\operatorname{sg}(\mu-1, \bar{v}-1)+p+1} s_{\mu-1} b_{q} \otimes\left(s_{\bar{v}-1}\left(a_{p} \otimes u_{p}\right) \cdot s_{\mu-1} v_{q}\right)\right) \\
= & \sum_{i=0}^{p+q+1} \sum_{(\bar{\mu}-1, v-1)}(-1)^{\operatorname{sg}(\bar{\mu}-1, v-1)}\left(\hat{\partial}^{p+q+1-i} s_{v-1} a_{p}\right. \\
& \left.\otimes \bar{f}_{h+h^{\prime}-1}\left(\partial_{0}^{i} s_{v-1} u_{p} \cdot \partial_{0}^{i} s_{\bar{\mu}-1}\left(b_{q} \otimes v_{q}\right)\right)\right) \\
& +\sum_{i=0}^{p+q+1}(-1)^{p+1} \sum_{(\mu-1, \bar{v}-1)}(-1)^{\operatorname{sg}(\mu-1, \bar{v}-1)}\left(\hat{\partial}^{p+q+1-i} s_{\mu-1} b_{q}\right. \\
& \left.\otimes \bar{f}_{h+h^{\prime}-1}\left(\partial_{0}^{i} s_{\bar{v}-1}\left(a_{p} \otimes u_{p}\right) \cdot \partial_{0}^{i} s_{\mu-1} v_{q}\right)\right) .
\end{aligned}
$$

At this point, we need to recall the same results from [6, pp. 57-58] which will play an essential role in the sequel. Let $(\alpha, \beta)$ be any $(p, q)$-shuffle and $i$ any integer with $0 \leq i \leq p+q$. The conditions

$$
\alpha_{r}<i, \quad \alpha_{r+1} \geq i ; \quad \beta_{t}<i, \quad \beta_{t+1} \geq i
$$

(or, $r=0$, if $\alpha_{1} \geq i ; r=p$, if $\alpha_{p}<i$, and similarly for $s$ ) uniquely determine a pair of integers $(r, t)$ satisfying:

$$
0 \leq r \leq p, \quad 0 \leq t \leq q, \quad r+t=i,
$$

and yield an $(r, t)$-shuffle

$$
\left(\alpha^{\prime}, \beta^{\prime}\right)=\left(\alpha_{1}, \ldots \alpha_{r} ; \beta_{1}, \ldots, \beta_{t}\right)
$$

and a $(p-r, q-t)$-shuffle

$$
\left(\alpha^{\prime \prime}, \beta^{\prime \prime}\right)=\left(\alpha_{r+1}-i, \ldots \alpha_{p}-i ; \beta_{t+1}-i, \ldots, \beta_{q}-i\right) .
$$

We then say that $(\alpha, \beta)$ has been split at $i$ into the two shuffles $\left(\alpha^{\prime}, \beta^{\prime}\right)$ and $\left(\alpha^{\prime \prime}, \beta^{\prime \prime}\right)$. Conversely, given integers $r, t$ as in (12), an $(r, t)$-shuffle $\left(\alpha^{\prime}, \beta^{\prime}\right)$, and a $(p-r, q-t)$ - 
shuffle $\left(\alpha^{\prime \prime}, \beta^{\prime \prime}\right)$, there exists a unique $(p, q)$-shuffle $(\alpha, \beta)$ which splits at $i=r+t$ into $\left(\alpha^{\prime}, \beta^{\prime}\right)$ and $\left(\alpha^{\prime \prime}, \beta^{\prime \prime}\right)$. The signatures of these shuffles are related by the equation

$$
\operatorname{sg}\left(\alpha^{\prime}, \beta^{\prime}\right)+\operatorname{sg}\left(\alpha^{\prime \prime}, \beta^{\prime \prime}\right)=\operatorname{sg}(\alpha, \beta)-t(p-r)
$$

If $(\alpha, \beta)$ is split at $i$, the face and degeneracy operators satisfy the following properties

$$
\begin{aligned}
\partial_{0}^{i} s_{\beta} & =s_{\beta^{\prime \prime}} \partial_{0}^{r} \quad \partial_{0}^{i} s_{\alpha}=s_{\alpha^{\prime \prime}} \partial_{0}^{t} \\
\hat{\partial}^{p+q-i} s_{\beta} & =s_{\beta^{\prime}} \hat{\partial}^{p-r} \quad \hat{\partial}^{p+q-i} s_{\alpha}=s_{\alpha^{\prime}} \hat{\partial}^{q-t} .
\end{aligned}
$$

Upon splitting each shuffle at $i,(10)$ and (11) can be rewritten as

$$
\begin{aligned}
& \sum_{i=0}^{p+q+1} \sum_{(\bar{\mu}-1, v-1)}(-1)^{\operatorname{sg}(\bar{\mu}-1, v-1)} s_{(v-1)^{\prime}} \hat{\partial}^{p-r} a_{p} \otimes \bar{f}_{h+h^{\prime}-1} \\
& \quad\left(s_{(v-1)^{\prime \prime}} \partial_{0}^{r} u_{p} \cdot s_{(\bar{\mu}-1)^{\prime \prime}} \partial_{0}^{t}\left(b_{q} \otimes v_{q}\right)\right) \\
& \quad+\sum_{i=0}^{p+q+1}(-1)^{p+1} \sum_{(\mu-1, \bar{v}-1)}(-1)^{\operatorname{sg}(\mu-1, \bar{v}-1)}\left(s_{(\mu-1)^{\prime}} \hat{\partial}^{q-t} b_{q}\right. \\
& \left.\otimes \bar{f}_{h+h^{\prime}-1}\left(s_{(\bar{v}-1)^{\prime \prime}} \partial_{0}^{r}\left(a_{p} \otimes u_{p}\right) \cdot s_{(\mu-1)^{\prime \prime}} \partial_{0}^{t} v_{q}\right)\right) .
\end{aligned}
$$

Let us observe that $s_{(v-1)^{\prime}} \hat{\partial}^{p-r} a_{p}$ appears in (14). The only case where it is a nondegenerate element in $\bar{K}$ is when $(v-1)^{\prime}$ is the empty set, i.e., $t=0$. In other words, it is the only summand which is not null modulo degeneracies. If $t=0$, then $\left((\bar{\mu}-1)^{\prime},(v-1)^{\prime}\right)$ is an $(r, 0)$-shuffle, and $\left((\bar{\mu}-1)^{\prime \prime},(v-1)^{\prime \prime}\right)$ is a $(p-r, q+1)$ shuffle. Moreover, by (13) we have $\operatorname{sg}\left((\bar{\mu}-1)^{\prime \prime},(v-1)^{\prime \prime}\right)=\operatorname{sg}(\bar{\mu}-1, v-1)$.

Thus, with the above results at hand, (14) can be rewritten as

$$
\begin{aligned}
& \sum_{r=0}^{p} \sum_{\left((\bar{\mu}-1)^{\prime \prime},(v-1)^{\prime \prime}\right)}(-1)^{\operatorname{sg}\left((\bar{\mu}-1)^{\prime \prime},(v-1)^{\prime \prime}\right)} \hat{\partial}^{p-r} a_{p} \otimes \bar{f}_{h+h^{\prime}-1}\left(s_{(v-1)^{\prime \prime}} \partial_{0}^{r} u_{p} \cdot s_{(\bar{\mu}-1)^{\prime \prime}}\left(b_{q} \otimes v_{q}\right)\right) \\
& =\sum_{r=0}^{p}\left[\hat{\partial}^{p-r} a_{p}\right] \mid\left[\overline { f } _ { h + h ^ { \prime } - 1 } \left(\sum_{\left((\bar{\mu}-1)^{\prime \prime},(v-1)^{\prime \prime}\right)}(-1)^{\operatorname{sg}\left((\bar{\mu}-1)^{\prime \prime},(v-1)^{\prime \prime}\right)}\right.\right. \\
& \left.\left.\quad \times s_{(v-1)^{\prime \prime}} \partial_{0}^{r} u_{p} \cdot s_{(\bar{\mu}-1)^{\prime \prime}}\left(b_{q} \otimes v_{q}\right)\right)\right] \\
& =\sum_{r=0}^{p}\left[\hat{\partial}^{p-r} a_{p}\right] \mid\left[\bar{f}_{h+h^{\prime}-1}\left(\partial_{0}^{r} u_{p} \nabla\left(b_{q} \otimes v_{q}\right)\right)\right] .
\end{aligned}
$$

Let us observe that $s_{(\mu-1)^{\prime}} \hat{\partial}^{q-t} b_{q}$ appears in (15). Once again, the only case that we have to study is for $r=0$; in this case, $\left((\mu-1)^{\prime},(\bar{v}-1)^{\prime}\right)$ is a $(0, t)$-shuffle, and $\left((\mu-1)^{\prime \prime},(\bar{v}-1)^{\prime \prime}\right)$ is a $(p+1, q-t)$-shuffle. Furthermore, $\operatorname{sg}(\mu-1, \bar{v}-1)=$ 
$\operatorname{sg}\left((\mu-1)^{\prime \prime},(\bar{v}-1)^{\prime \prime}\right)+t(p+1)$. Hence (15) becomes

$$
\begin{aligned}
& \sum_{t=0}^{q}(-1)^{p+1} \sum_{\left((\mu-1)^{\prime \prime},(\bar{v}-1)^{\prime \prime}\right)}(-1)^{\left.\operatorname{sg}\left((\mu-1)^{\prime \prime},(\bar{v}-1)^{\prime \prime}\right)\right)+t(p+1)} \\
& \times\left(\hat{\partial}^{q-t} b_{q} \otimes \bar{f}_{h+h^{\prime}-1}\left(s_{(\bar{v}-1)^{\prime \prime}}\left(a_{p} \otimes u_{p}\right) \cdot s_{(\mu-1)^{\prime \prime}} \partial_{0}^{t} v_{q}\right)\right) \\
& =\sum_{t=0}^{q}(-1)^{(p+1)(t+1)}\left[\hat{\partial}^{q-t} b_{q}\right] \mid\left[\overline { f } _ { h + h ^ { \prime } - 1 } \left(\sum_{\left((\mu-1)^{\prime \prime},(\bar{v}-1)^{\prime \prime}\right)}(-1)^{\left.\operatorname{sg}\left((\mu-1)^{\prime \prime},(\bar{v}-1)^{\prime \prime}\right)\right)}\right.\right. \\
& \left.\left.\times s_{\bar{v}-1)^{\prime \prime}}\left(a_{p} \otimes u_{p}\right) \cdot s_{(\mu-1)^{\prime \prime}} \partial_{0}^{t} v_{q}\right)\right] \\
& =\sum_{t=0}^{q}(-1)^{(p+1)(t+1)}\left[\hat{\partial}^{q-t} b_{q}\right] \mid\left[\bar{f}_{h+h^{\prime}-1}\left(\left(a_{p} \otimes u_{p}\right) \nabla \partial_{0}^{t} v_{q}\right)\right]
\end{aligned}
$$

The terms (16) and (6) are equal to each other; analogously, for (17) and (7). Hence, we can state that $\bar{f}_{k}$ is multiplicative for each non-negative integer $k$, and $\bigoplus_{k \geq 0} \bar{f}_{k}$ is a morphism of DGA-algebras.

Secondly, the inclusion $\bigoplus_{k \geq 0} \tilde{g}_{k}$ is a DGA-algebra morphism, since EilenbergMac Lane proved in [5] that $g_{\bar{W}-\bar{B}}$ is a DGA-algebra morphism and as was seen in the previous section $g_{\bar{W}-\bar{B}}=\bigoplus_{k \geq 0} \tilde{g}_{k}$.

Thirdly, we are going to show that $\tilde{\phi}_{k}$ is a quasi algebra homotopy, for each non-negative integer $k$. That is,

$$
\bar{\phi}_{k} \nabla\left(\bar{\phi}_{k-i} \otimes \bar{g}_{i}\right)=0, \quad \bar{\phi}_{k} \nabla\left(\bar{g}_{k-i} \otimes \bar{\phi}_{i}\right)=0, \quad \bar{\phi}_{k} \nabla\left(\bar{\phi}_{k-i} \otimes \bar{\phi}_{i}\right)=0 .
$$

Now, we make some useful observations to prove the desired result:

1. We will use the fact that the Eilenberg-Zilber contraction, $E Z_{A, A^{\prime}}:\left\{\left(A \times A^{\prime}\right)_{N}\right.$, $A_{N} \otimes A_{N}^{\prime}, A W_{A, A^{\prime}}, E M L_{A, A^{\prime}}, S H I_{A, A^{\prime}}$ \} is a semi-full algebra contraction where $A$ and $A^{\prime}$ are commutative simplicial algebras (see [1]);

2. From the definition of $E M L$, it follows that

$$
1_{p} \times a_{p}=E M L\left(1_{0} \otimes a_{p}\right)
$$

3. A property involving the different products that appear in our setting:

$$
\begin{aligned}
\left(a_{p} \otimes u_{p}\right) \nabla\left(b_{q} \otimes v_{q}\right)= & \left(a_{p} \otimes u_{p}\right) \nabla^{\prime}\left(1_{q+1} \otimes b_{q} \otimes v_{q}\right) \\
& +(-1)^{p+1}\left(1_{p+1} \otimes a_{p} \otimes u_{p}\right) \nabla^{\prime}\left(b_{q} \otimes v_{q}\right) \\
= & \left(a_{p} \times u_{p}\right) \bigcirc\left(1_{q+1} \times\left(b_{q} \otimes v_{q}\right)\right) \\
& +(-1)^{p+1}\left(1_{p+1} \times\left(a_{p} \otimes u_{p}\right)\right) \bigcirc\left(b_{q} \times v_{q}\right),
\end{aligned}
$$

where $\odot$ denotes the external product in $K \times \bar{W}(K)$. Here we use

$$
c_{p} \diamond d_{q}=c_{p} \diamond^{\prime} s_{0} d_{q}+(-1)^{p} s_{0} c_{p} \diamond^{\prime} d_{q},
$$


where $c_{p} \in A_{p}, d_{q} \in A_{q}, A$ is a simplicial algebra, $\diamond$ is the external product over $A$ and $\diamond^{\prime}$ is its derived product (see [5, (6.10) p. 71]).

Remark 4.3. Let us observe that $\bar{K} \times\left(\bigoplus_{k \geq 0} \widetilde{W}^{(k)}(K)\right)$ is an ideal of $K \times \bar{W}(K)$ with regards to the inner product and the external product.

4. Let us recall that the product on $\bar{K} \otimes\left(\bigoplus_{k \geq 0} \widetilde{W}^{(k)}(K)\right)$ is $(\diamond \otimes \nabla)(1 \otimes T \otimes 1)$, where $T(a \otimes b)=(-1)^{|a||b|} b \otimes a$.

We begin by proving that

$$
\bar{\phi}_{k} \nabla\left(\bar{\phi}_{k-i} \otimes \bar{g}_{i}\right)=0 \quad \text { for all } k \geq 0 .
$$

We proceed by induction on $k$.

If $k=0$ or $k=1$, then the identity (20) is immediate, since $\tilde{\phi}_{0}$ and $\tilde{\phi}_{1}$ are both null morphisms. Assume the formula (20) holds for $k \leq n$; we will prove it for $k=n$. Suppose that $n=h+h^{\prime}$, where $h$ y $h^{\prime}$ are non-negative integers, $a_{p} \otimes$ $u_{p} \in \widetilde{W}_{p+1, N}^{(h)}(K)$ and $\left[b_{q}\right] \mid v \in \widetilde{B}_{l, N}^{\left(h^{\prime}\right)}\left(K_{N}\right)$ with $a_{p} \in \bar{K}_{p}$ and $b_{q} \in \bar{K}_{q}$. Computing the left term of the identity $(20)$ on $\left(a_{p} \otimes u_{p}\right) \otimes\left(\left[b_{q}\right] \mid v\right)$. We have:

$$
\bar{\phi}_{n}\left(\bar{\phi}_{h}\left(a_{p} \otimes u_{p}\right) \nabla \bar{g}_{h^{\prime}}\left(\left[b_{q}\right] \mid v\right)\right)
$$

(using (19) and the definitions of the morphisms, the above expression can be rewritten)

$$
\begin{aligned}
= & \left(-S H I_{n-1}-E M L_{n-1}\left(1 \otimes \bar{\phi}_{n-1}\right) A W_{n-1}\right)(-1)^{p+2} \\
& \times\left(\left(1_{p+2} \times \bar{\phi}_{h}\left(a_{p} \otimes u_{p}\right)\right) \odot E M L_{h^{\prime}-1}\left(1 \otimes \bar{g}_{h^{\prime}-1}\right)\left(b_{q} \otimes v\right)\right) \\
& +\left(-S H I_{n-1}-E M L_{n-1}\left(1 \otimes \bar{\phi}_{n-1}\right) A W_{n-1}\right) \\
& \times\left(\bar{\phi}_{h}\left(a_{p} \otimes u_{p}\right) \oslash\left(1_{l} \times E M L_{h^{\prime}-1}\left(1 \otimes \bar{g}_{h^{\prime}-1}\right)\left(b_{q} \otimes v\right)\right)\right)
\end{aligned}
$$

(expanding both summands)

$$
\begin{aligned}
= & (-1)^{p+1} S H I_{n-1}\left(\left(1_{p+2} \times \bar{\phi}_{h}\left(a_{p} \otimes u_{p}\right)\right) \odot E M L_{h^{\prime}-1}\left(1 \otimes \bar{g}_{h^{\prime}-1}\right)\left(b_{q} \otimes v\right)\right) \\
& +(-1)^{p+1}\left(E M L_{n-1}\left(1 \otimes \bar{\phi}_{n-1}\right) A W_{n-1}\right) \\
& \times\left(\left(1_{p+2} \times \bar{\phi}_{h}\left(a_{p} \otimes u_{p}\right)\right) \odot E M L_{h^{\prime}-1}\left(1 \otimes \bar{g}_{h^{\prime}-1}\right)\left(b_{q} \otimes v\right)\right) \\
- & S H I_{n-1}\left(\bar{\phi}_{h}\left(a_{p} \otimes u_{p}\right) \bigcirc\left(1_{l} \times E M L_{h^{\prime}-1}\left(1 \otimes \bar{g}_{h^{\prime}-1}\right)\left(b_{q} \otimes v\right)\right)\right) \\
- & E M L_{n-1}\left(1 \otimes \bar{\phi}_{n-1}\right) A W_{n-1}\left(\bar{\phi}_{h}\left(a_{p} \otimes u_{p}\right)\right. \\
& \left.\bigotimes\left(1_{l} \times E M L_{h^{\prime}-1}\left(1 \otimes \bar{g}_{h^{\prime}-1}\right)\left(b_{q} \otimes v\right)\right)\right) .
\end{aligned}
$$

In the sequel, we shall prove that each of the summands are zero.

Using (18), we have

$$
\left(1_{p+2} \times \bar{\phi}_{h}\left(a_{p} \otimes u_{p}\right)\right)=E M L_{h}\left(1_{0} \otimes \bar{\phi}_{h}\left(a_{p} \otimes u_{p}\right)\right) .
$$


Taking into account the above identity, the summand (22) can be written as

$$
(-1)^{p+1} S H I_{n-1}\left(E M L_{h}\left(1_{0} \otimes \bar{\phi}_{h}\left(a_{p} \otimes u_{p}\right)\right) \odot E M L_{h^{\prime}-1}\left(1 \otimes \bar{g}_{h^{\prime}-1}\right)\left(b_{q} \otimes v\right)\right)
$$

(using the fact that EML is a morphism of DGA-algebras, then, up to sign)

$$
\left.=S H I_{n-1} E M L_{n-1}\left(\left(1_{0} \diamond b_{q}\right) \otimes\left(\bar{\phi}_{h}\left(a_{p} \otimes u_{p}\right) \nabla \bar{g}_{h^{\prime}-1}\right)(v)\right)\right)=0
$$

since $S H I_{n-1} E M L_{n-1}=0$.

Let us prove that (23) vanishes. By (26), (23) can be written as

$$
\begin{aligned}
& (-1)^{p+1} E M L_{n-1}\left(1 \otimes \bar{\phi}_{n-1}\right) A W_{n-1} \\
& \quad \times\left(E M L_{h}\left(1_{0} \otimes \bar{\phi}_{h}\left(a_{p} \otimes u_{p}\right)\right) \subseteq E M L_{h^{\prime}-1}\left(1 \otimes \bar{g}_{h^{\prime}-1}\right)\left(b_{q} \otimes v\right)\right)
\end{aligned}
$$

(taking into account that $E M L$ is a morphism of DGA-algebras and $A W_{n-1} E M L_{n-1}$ is the identity morphism, up to sign)

$$
=E M L_{n-1}\left(1_{0} \diamond b_{q} \otimes \bar{\phi}_{n-1}\left(\bar{\phi}_{h}\left(a_{p} \otimes u_{p}\right) \nabla \bar{g}_{h^{\prime}-1}(v)\right)\right)=0,
$$

(since by the inductive assumption $\bar{\phi}_{n-1}\left(\bar{\phi}_{h}\left(a_{p} \otimes u_{p}\right) \nabla \bar{g}_{h^{\prime}-1}(v)\right)=0$ ).

On the other hand, using (18)

$$
1_{l} \times E M L_{h^{\prime}-1}\left(1 \otimes \bar{g}_{h^{\prime}-1}\right)\left(b_{q} \otimes v\right)=E M L_{h^{\prime}}\left(1_{0} \otimes E M L_{h^{\prime}-1}\left(1 \otimes \bar{g}_{h^{\prime}-1}\right)\left(b_{q} \otimes v\right)\right) .
$$

Hence (24) may be rewritten as

$$
-\operatorname{SHI}_{n-1}\left(\bar{\phi}_{h}\left(a_{p} \otimes u_{p}\right) \oslash E M L_{h^{\prime}}\left(1_{0} \otimes E M L_{h^{\prime}-1}\left(1 \otimes \bar{g}_{h^{\prime}-1}\right)\left(b_{q} \otimes v\right)\right)\right)
$$

(by definition of $\bar{\phi}_{h}$ )

$$
\begin{aligned}
= & -S H I_{n-1}\left(S H I_{h-1}\left(a_{p} \otimes u_{p}\right) \triangle E M L_{h^{\prime}}\left(1_{0} \otimes E M L_{h^{\prime}-1}\left(1 \otimes \bar{g}_{h^{\prime}-1}\right)\left(b_{q} \otimes v\right)\right)\right) \\
- & S H I_{n-1}\left(E M L_{h-1}\left(1 \otimes \bar{\phi}_{h-1}\right) A W_{h-1}\left(a_{p} \otimes u_{p}\right)\right. \\
& \left.\varnothing E M L_{h^{\prime}}\left(1_{0} \otimes E M L_{h^{\prime}-1}\left(1 \otimes \bar{g}_{h^{\prime}-1}\right)\left(b_{q} \otimes v\right)\right)\right) .
\end{aligned}
$$

Using the fact that $S H I_{n-1}$ is a quasi algebra homotopy, we conclude that the first summand is zero. The second one vanishes as well, since $E M L$ is a morphism of DGA-algebras and $S H I_{n-1} E M L_{n-1}=0$. Thereby (24) vanishes.

Finally, let us show that (25) vanishes as well. In stating (27), we can write (25) as

$$
-E M L_{n-1}\left(1 \otimes \bar{\phi}_{n-1}\right) A W_{n-1}\left(\bar{\phi}_{h}\left(a_{p} \otimes u_{p}\right) \odot E M L_{h^{\prime}}\left(1_{0} \otimes E M L_{h^{\prime}-1}\left(1 \otimes \bar{g}_{h^{\prime}-1}\right)\left(b_{q} \otimes v\right)\right)\right)
$$

(by the definition of $\bar{\phi}_{h}$ )

$$
\begin{aligned}
= & -E M L_{n-1}\left(1 \otimes \bar{\phi}_{n-1}\right) A W_{n-1} \\
& \times\left(S H I_{h-1}\left(a_{p} \otimes u_{p}\right) \odot E M L_{h^{\prime}}\left(1_{0} \otimes E M L_{h^{\prime}-1}\left(1 \otimes \bar{g}_{h^{\prime}-1}\right)\left(b_{q} \otimes v\right)\right)\right)
\end{aligned}
$$




$$
\begin{aligned}
& -E M L_{n-1}\left(1 \otimes \bar{\phi}_{n-1}\right) A W_{n-1} \\
& \times\left(E M L_{h-1}\left(1 \otimes \bar{\phi}_{h-1}\right) A W_{h-1}\left(a_{p} \otimes u_{p}\right)\right. \\
& \left.\triangle E M L_{h^{\prime}}\left(1_{0} \otimes E M L_{h^{\prime}-1}\left(1 \otimes \bar{g}_{h^{\prime}-1}\right)\left(b_{q} \otimes v\right)\right)\right) .
\end{aligned}
$$

The first summand is zero since

$$
A W_{n-1}\left(S H I_{h-1} \odot E M L_{h^{\prime}}\right)=0 .
$$

This identity is deduced from the following facts, $A W$ is a DGA-algebra morphism and $A W S H I=0$.

Taking into account that $E M L$ is a morphism of DGA-algebras and $A W_{n-1} E M L_{n-1}$ is the identity morphism, the second summand can be rewritten, up to sign, as

$$
\sum_{i=0}^{p} E M L_{n-1}\left(( \hat { \partial } ^ { p - i } a _ { p } \diamond 1 _ { 0 } ) \otimes \left(\bar{\phi}_{n-1}\left(\bar{\phi}_{h-1}\left(\partial_{0}^{i} u_{p}\right) \nabla \bar{g}_{h^{\prime}}\left(b_{q} \otimes v\right)\right)\right.\right.
$$

But this summand is zero since $\bar{\phi}_{n-1} \nabla\left(\bar{\phi}_{h-1} \otimes \bar{g}_{h^{\prime}}\right)=0$, by the inductive hypothesis. This concludes the proof of (20).

The second identity,

$$
\bar{\phi}_{k} \nabla\left(\bar{g}_{k-i} \otimes \bar{\phi}_{i}\right)=0
$$

is evident since $\nabla$ is commutative; therefore, both identities (28) and (20) are equal to each other. Finally, the third identity,

$$
\bar{\phi}_{k} \nabla\left(\bar{\phi}_{k-i} \otimes \bar{\phi}_{i}\right)=0
$$

may be proven in an analogous way to (20). Hence, $\bigoplus_{k \geq 0} \tilde{\phi}_{k}$ is a quasi algebra homotopy.

Theorem 4.4. The contraction, $C_{\bar{W}-\bar{B}}:\left\{\bar{W}_{N}(K), \bar{B}_{N}\left(K_{N}\right), f_{\bar{W}-\bar{B}}, g_{\bar{W}-\bar{B}}, \phi_{\bar{W}-\bar{B}}\right\}$, is a semi-full algebra contraction.

Proof. a) Theorem 4.2 states that $\sum_{k \geq 0} r^{(k)}$ is an almost-full algebra contraction, and at the beginning of this section we proved that the morphism $\rho$ is a derivation.

b) This $\sum_{k \geq 0} r^{(k)}$ contraction, can be perturbed into $C_{\bar{W}-\bar{B}}$ taking $\rho$ as a perturbation datum.

Hence, using Theorem 4.1, we obtain the desired conclusion.

\section{HOMOLOGY OF TWISTED CARTESIAN PRODUCTS OF EILENBERG-MAC LANE SPACES}

We find that a remarkable consequence of Theorem 4.4 in the computation of the homology of Eilenberg-Mac Lane spaces, $K(\pi, n)$, where $\pi$ is an abelian 
group and $n$ is a non-negative integer. In the sequel, we follow the iterative simplicial description of $K(\pi, n)$ in terms of the classifying complex $(W)$ given in [14, Theorem 23.2]. Taking as a starting point Eilenberg-Mac Lane's work in $[5,6]$, Real states in [15] a contraction $C_{\pi, n}$ from the normalized chain complex $C_{N}(K(\pi, n))$ canonically associated to $K(\pi, n)$ to a tensor product of Cartan's elementary complexes (see [4]), denoted by $G(n, \pi)$. For this purpose, the following chain of contractions is established:

$$
C_{N}(K(\pi, 1)) \Rightarrow \bar{W}_{N}(C(K(\pi, 0))) \Rightarrow \bar{B}_{N}(\Lambda(\pi)) \Rightarrow G(\pi, 1)
$$

Using the iterative simplicial description of $K(\pi, n)$, we have

$$
\begin{aligned}
C_{N}(K(\pi, n+1)) & \Rightarrow \bar{W}_{N}\left(\bar{W}^{n}(C(K(\pi, 0)))\right) \Rightarrow \bar{B}_{N}\left(\bar{W}_{N}^{n}(C(K(\pi, 0)))\right) \\
& \Rightarrow \bar{B}_{N}\left(\bar{B}_{N}^{n}(\Lambda(\pi))\right) \Rightarrow \bar{B}_{N}(G(\pi, n)) \Rightarrow G(\pi, n+1) .
\end{aligned}
$$

In order to establish the last contraction of the above chain, Real needed to fix $\mathbf{Z}$ localised over a prime $p$ as ground ring. In [16] it is proven that the last three contractions are, at least, semi-full algebra contractions. Now, taking into account that the first one is an algebra isomorphism (see [10, p. 81]), and using Theorem 4.4 we showed that all the contractions involved in the precedent chain are semi-full algebra contractions. Hence we can state the following result.

Theorem 5.1. Taking $\mathbf{Z}$ localised over a prime $p$ as ground ring, given an abelian group $\pi$ and a non-negative integer $n$. There exists a semi-full algebra contraction, $C_{\pi, n}$, from $C_{N}(K(\pi, n))$ to $G(\pi, n)$.

Now, we try to take advantage of this result for achieving economical homological computations of some fiber bundles. To this end, we need the following theorem.

Theorem 5.2 [1]. Let $G^{\prime \prime}$ be a simplicial group operating on the left of $G$ and $\tau$ : $G^{\prime} \rightarrow G^{\prime \prime}$ be a twisting function such that the twisted cartesian product $(T C P) G \times{ }_{\tau}$ $G^{\prime}$ is a simplicial group with regards to the trivial group multiplication. Then, the contraction associated to $G \times{ }_{\tau} G^{\prime}$ by applying Theorem 2.2 is a semi-full algebra contraction.

It is well known (see [14, Lemma 25.1]) that if $f: \pi \rightarrow \pi^{\prime}$ is a homomorphism of groups, then $f$ induces a homomorphism of groups between $K(\pi, n)$ and $K\left(\pi^{\prime}, n\right)$, which we denote by $f$ as well. It is apparent that if $f: \pi \rightarrow \pi^{\prime}$ is a homomorphism of groups, then the twisted cartesian product $E_{f}=K(\pi, n) \times_{\tau_{u} \circ f} K\left(\pi^{\prime}, n+1\right)$ induced by $f$ from the universal (principal) TCP $K(\pi, n) \times_{\tau_{u}} K(\pi, n+1)$ is a simplicial group. Then, $E_{f}$ provides examples of TCP satisfying the hypothesis of Theorem 5.2.

Combining both Theorems 5.1 and 5.2 we conclude the following proposition.

Proposition 5.3. Let $E=K(\pi, n) \times{ }_{\tau} K\left(\pi^{\prime}, n^{\prime}\right)$ be a TCP, where the structural group is reduced and working in $\mathbf{Z}$ localised over a prime $p$ as ground ring. If $E$ is a group 
with regards to the group multiplication, then there exists a semi-full algebra contraction from

$$
C_{N}\left(K(\pi, n) \times{ }_{\tau} K\left(\pi^{\prime}, n^{\prime}\right)\right)
$$

to a twisting tensor product $G(\pi, n) \tilde{\otimes} G\left(\pi^{\prime}, n^{\prime}\right)$.

Proof. We only give the main ideas of the proof. By Theorem 5.2, we have the following semi-full algebra contraction:

$$
C_{N}(E) \Rightarrow C_{N}(K(\pi, n)) \otimes_{t} C_{N}\left(K\left(\pi, n^{\prime}\right)\right)
$$

Furthermore, $C_{N}(K(\pi, n)) \otimes_{t} C_{N}\left(K\left(\pi, n^{\prime}\right)\right)$ is DGA-algebra with regards to the natural product of $C_{N}(K(\pi, n)) \otimes C_{N}\left(K\left(\pi^{\prime}, n^{\prime}\right)\right)$. Thus $t \cap$ is a derivation.

Using Theorem 5.1 and the tensor product of contractions, we can establish the following semi-full algebra contraction:

$$
C_{N}(K(\pi, n)) \otimes C_{N}\left(K\left(\pi^{\prime}, n^{\prime}\right)\right) \Rightarrow G(\pi, n) \otimes G\left(\pi^{\prime}, n^{\prime}\right)
$$

Taking $t \cap$ as the perturbation datum, this contraction can be perturbed into

$$
C_{N}(K(\pi, n)) \otimes_{t} C_{N}\left(K\left(\pi^{\prime}, n^{\prime}\right)\right) \Rightarrow\left(G(\pi, n) \otimes G\left(\pi^{\prime}, n^{\prime}\right), d^{\otimes}+d_{t \cap}\right) .
$$

The pointwise nilpotency condition is guaranteed (see [12, Lemma 3.4.]) using the standard filtration on $C_{N}(K(\pi, n)) \otimes C_{N}\left(K\left(\pi^{\prime}, n^{\prime}\right)\right)$. Once the structural group is reduced, $t$ vanishes on one-simplices (see [14]).

Finally, composing contractions (29) and (30), the desired result is concluded.

In the following example (extracted from [1]) we show how it is possible to take advantage of the multiplicative properties of the chain complexes in order to compute in an economical way homological models for some TCP of two Eilenberg-Mac Lane spaces.

Example 5.4. Let $E=K\left(\mathbf{Z}_{2}, 1\right) \times_{\tau_{u} f} K(\mathbf{Z}, 2)$ be the principal TCP induced from $K\left(\mathbf{Z}_{2}, 1\right) \times_{\tau_{u}} K\left(\mathbf{Z}_{2}, 2\right)$ by $f: \mathbf{Z} \rightarrow \mathbf{Z}_{2}$. It is clair that $E$ satisfies the hypotheses of Proposition 5.3. Fix $\mathbf{Z}$ as ground ring. Combining the results of [6] and [16] it is possible to establish the following semi-full algebra contraction

$$
C_{N}\left(K\left(\mathbf{Z}_{2}, 1\right)\right) \Rightarrow E\left(u_{1}, 1\right) \otimes \Gamma\left(v_{1}, 2\right),
$$

where $d\left(\gamma_{j}\left(v_{1}\right)\right)=2 u_{1} \gamma_{j-1}\left(v_{1}\right)$. (It is, actually, almost-full.)

And

$$
C_{N}(K(\mathbf{Z}, 2)) \Rightarrow \Gamma\left(v_{2}, 2\right)
$$


Here $E(u, 1)$ denotes the exterior algebra with one generator $u$ in degree 1 , and $\Gamma(v, 2)$ denotes the divided power algebra with on generator $v$ in degree 2 . In this case, the contraction (30) can be written as

$$
C_{N}\left(K\left(\mathbf{Z}_{2}, 1\right)\right) \otimes_{t} C_{N}(K(\mathbf{Z}, 2)) \Rightarrow\left(\left(E\left(u_{1}, 1\right) \otimes \Gamma\left(v_{1}, 2\right)\right) \otimes \Gamma\left(v_{2}, 2\right), d^{\otimes}+d_{t \cap}\right)
$$

Let us observe that the smaller complex of this contraction is a DGA-algebra with regards to the same product of $\left(\left(E\left(u_{1}, 1\right) \otimes \Gamma\left(v_{1}, 2\right)\right) \otimes \Gamma\left(v_{2}, 2\right), d^{\otimes}\right)$. Thus, $d_{t \cap}$ is a derivation. This fact has important consequences in order to compute $d_{t \cap}$ on the whole set of additive generators of the homological model (as a module). By Basic Perturbation Lemma, we have

$$
d_{t \cap}=f(t \cap) \Sigma_{c}^{t \cap} g
$$

By direct computation, we obtain

$$
d_{t \cap}\left(\gamma_{1}\left(v_{2}\right)\right)=u_{1} .
$$

Taking into account that

$$
\gamma_{i}\left(v_{2}\right) \gamma_{j}\left(v_{2}\right)=\left(\begin{array}{c}
i+j \\
i
\end{array}\right) \gamma_{i+j}\left(v_{2}\right)
$$

and that $d_{t \cap}$ is a derivation, we have

$$
d_{t \cap}\left(\gamma_{j}\left(v_{2}\right)\right)=u_{1} \gamma_{j-1}\left(v_{2}\right)
$$

By a similar argument, we can calculate $d_{t \cap}$ on any additive generator of the module; in this way, it is easy to see that $d_{t \cap}$ is null on all additive generators except on $\gamma_{j}\left(v_{2}\right)$.

Summing up, in order to compute $d_{t \cap}$ over all additive generators of the module, we only use the formula (31) over the generators of the algebra: $u_{1}, v_{1}$, and $v_{2}$. For the remainder of the additive generators we use the multiplicative property of $d_{t \cap}$. Let us observe that this represents an economical way for computing $d_{t \cap}$, alternative to the formula (31).

\section{CONCLUSIONS AND FURTHER WORK}

In this article, we presented a technique that improves the computation of the homology of certain twisted cartesian products of two Eilenberg-Mac Lane spaces.

One of our next goals is to find a manageable formula for the projection of the contraction between $\bar{W}_{N}(K)$ and $\bar{B}_{N}\left(K_{N}\right)$. This formula will allow us to compute a cohomology class $\zeta \in H^{m}(K(\pi, n), G)$ (we have exploited this idea in [2] to obtain a generating set for the second cohomology group in terms of representative 2 -cocycles over a group $\pi$ ). These cohomology classes can define the twisted cartesian products of two Eilenberg-Mac Lane spaces, $K(G, m-1) \times_{\tau(\zeta)}$ $K(\pi, n)$ (see [14, Lemmas 21.9 and 24.3]). Another of our goals is to study the algebra structure on this kind of twisted cartesian products of Eilengerg-Mac Lane spaces since they might be an interesting source of examples where we can apply our technique. 


\section{ACKNOWLEDGMENT}

We would like to thank Kristeen Cheng for her reading of this article. This work was partially supported by the PAICYT research project FQM-296 from Junta de Andalucía (Spain).

\section{REFERENCES}

[1] Álvarez, V., Armario, J. A., Frau, M. D., Real, P. (2007). Algebra structures on the twisted Eilenberg-Zilber theorem. Communications in Algebra 35:3273-3291.

[2] Álvarez, V., Armario, J. A., Frau, M. D., Real, P. (2009). The homological reduction method for computing cocyclic Hadamard matrices. Journal of Symbolic Computation 44.

[3] Brown, R. (1967). The twisted Eilenberg-Zilber theorem. Celebrazioni Archimedee del secolo XX. Simposio di topologia 33-37.

[4] Cartan, H. (1956). Algèbres d'Eilenberg-Mac Lane. Séminaire H. Cartan 1954/55, (expos é 2 à 11). Ecole Normale Superieure, Paris.

[5] Eilenberg, S., Mac Lane, S. (1953). On the groups $H(\pi, n)$, I. Annals of Math. 58:55-106.

[6] Eilenberg, S., Mac Lane, S. (1954). On the groups $H(\pi, n)$, II. Annals of Math. 66:49-149.

[7] Eilenberg, S., Zilber, J. A. (1953). On products of complexes. Am. J. Math. 75:200-204.

[8] Gugenheim, V. K. A. M., Lambe, L. A. (1989). Perturbation theory in differential homological algebra I. Illinois J. Math. 33(4):566-582.

[9] Gugenheim, V. K. A. M., Lambe, L. A., Stasheff, J. D. (1991). Perturbation theory in differential homological algebra II. Illinois J. Math. 35(3):357-373.

[10] Gugenheim, V. K. A. M., May, J. P. (1974). On the theory and application of differential torsion products. Memo. Amer. Math. Soc. 142.

[11] Huebschmann, J., Kadeishvili, T. (1991). Small models for chain algebras. Math. Z. 209:245-280.

[12] Lambe, L. A., Stasheff, J. D. (1987). Applications of perturbation theory to iterated fibrations. Manuscripta Math. 58:367-376.

[13] Mac Lane, S. (1995). Homology. Classics in Mathematics. Berlin. Springer-Verlag: Reprint of the 1975 edition.

[14] May, J. P. (1967). Simplicial Objects in Algebraic Topology. Princeton, NJ: Van Nostrad.

[15] Real, P. (1993). Algorithms for Computing the Effective Homology of Classifying Spaces. Ph.D. thesis. University of Seville. Seville, Spain.

[16] Real, P. (2000). Homological perturbation theory and associativity. Homology, Homotopy and Applications 2:51-88.

[17] Real, P., Sergeraert, F. (1994). A conjecture of Eilenberg-Mac Lane. Institut Fourier (Preprint).

[18] Shih, W. (1962). Homologie des espaces fibrés. Inst. Hautes Etudes Sci. 13:93-176.

[19] Wong, S. C. (1985). Comparison Between the Reduced Bar Construction and the Reduced W-Construction. Diplomarbeit. Math. Institut der Universität Heidelberg, Heidelberg, Germany. 